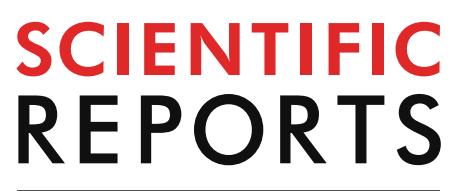

natureresearch

Check for updates

\title{
OPEN Ultra-compact high efficiency and low crosstalk optical interconnection structures based on inverse designed nanophotonic elements
}

\begin{abstract}
Zikang $\mathrm{Li}^{1,2}{ }^{2}$, Guofeng $\mathrm{Li}^{1}$, Jie Huang ${ }^{2}$, Zhenrong Zhang ${ }^{1 凶}$, Junbo Yang ${ }^{2,3 \bowtie}$, Changming Yang ${ }^{1}$, Yang Oian ${ }^{1}$, Wenjie $X_{u^{1}}$ \& Huimin Huang ${ }^{1}$

In this paper, we combine inverse design concept and direct binary search algorithm to demonstrate three ultra-compact high efficiency and low crosstalk on-chip integrated optical interconnection basic devices in the entire wavelength range of 1,400-1600 $\mathrm{nm}$ based on silicon-on-insulator platform. A 90-degree waveguide bend with a footprint of only $2.4 \times 2.4 \mu^{2}$ is designed, whose transmission efficiency up to $0.18 \mathrm{~dB}$. A waveguide crossing with a footprint of only $2.4 \times 2.4 \mu \mathrm{m}^{2}$ is designed, which can provide insertion loss of less than $0.5 \mathrm{~dB}$ and crosstalk (CL) of lower than $-19 \mathrm{~dB}$. A same direction waveguide crossing with footprint of only $2.4 \times 3.6 \mu \mathrm{m}^{2}$ is designed, which can provide the insertion loss of less than $0.56 \mathrm{~dB}$ and the crosstalk of lower than $-21 \mathrm{~dB}$. Then, we use them to form several ultra-compact optical interconnect basic structures and performed the simulation calculation. They overall achieve high performance. This will significantly improve the integration density.
\end{abstract}

The concept of integrated photonics was introduced in 1969 by Miller ${ }^{1}$, which made a great contribution to the on-chip integrated optical interconnection. On-chip integrated optical interconnection is an emerging technique for large capacity data communications. The waveguide bends and crossings are one of the most critical components of optical interconnection. There are many waveguide bends and crossings designed by conventional approaches. A $5 \mu \mathrm{m}$ radius 90 -degree circular bend with $400 \times 220 \mathrm{~nm}$ silicon strip waveguide was designed ${ }^{2}$. Although the loss of the circular bend is $0.023 \mathrm{~dB}$ in FDTD simulation, the design method need a lot of complicated artificial adjustment experiments. A 90-degree partial Euler bends with an effective radius of $50 \mu \mathrm{m}$ was fabricated on a silicon nitride photonic ${ }^{3}$. The bend loss was within $0.2 \mathrm{~dB}$, but it was only limited to the wavelength of $850 \mathrm{~nm}$ and had a large footprint. A $275 \mu \mathrm{m}$ radius circular bend was designed using a silicon waveguide ${ }^{4}$, which achieved very low loss. Unfortunately the length of the radius and waveguide is too long. Bogaerts et al. ${ }^{5}$ proposed a 90 -degree adiabatic bend for silicon waveguides. The total bend loss was $0.001 \mathrm{~dB}$. However, the curve radius and circular wire section were up to $5 \mu \mathrm{m}$ and $25 \mu \mathrm{m}$, respectively. Other waveguide bends with $2 \mu \mathrm{m}^{6}, 4 \mu \mathrm{m}^{7}$, $10 \mu \mathrm{m}^{8}$ radius were designed using a straight waveguide. Although the loss was below $0.3 \mathrm{~dB}$ loss, they had a large footprint and need a very long waveguide. Various studies have also been conducted on waveguide crossings. A low-loss waveguide crossing using the self-imaging properties of multimode interference (MMI) structures was designed ${ }^{9}$, which achieved a loss of only $0.009 \mathrm{~dB}$. But, the length is also fairly long (>140 $\mu \mathrm{m}$ in the case). A multimode-interference (MMI)-based crossing in high-index-contrast silicon wire waveguides was reported, which had an MMI crossing of $\sim 0.4 \mathrm{~dB}$ insertion loss ${ }^{10}$. However, its large $13 \times 13 \mu \mathrm{m}^{2}$ footprint may be an issue for dense integration. Recently there have been reports attempting to further reduce the footprint with successful demonstrations with a $6 \times 6 \mu \mathrm{m}^{2}{ }^{11-14}$. Note that $-1.7 \mathrm{~dB}$ insertion loss was achieved with only $-40 \mathrm{~dB}$ cross

\footnotetext{
${ }^{1}$ Guangxi Key Laboratory of Multimedia Communications and Network Technology, School of Computer, Electronics and Information, Guangxi University, Nanning 530004, China. ${ }^{2}$ Center of Material Science, National University of Defense Technology, Changsha 410073, China. ${ }^{3}$ State Key Laboratory on Advanced Optical Communication Systems and Networks, Peking University, Beijing 100871, China. ${ }^{4}$ These authors contributed equally: Zikang Li and Guofeng Li. ${ }^{\circledR}$ email: zzr76@gxu.edu.cn; yangjunbo@nudt.edu.cn
} 
talk ${ }^{11}$. Insertion loss was lower than $0.2 \mathrm{~dB}$ and crosstalk loss was below $40 \mathrm{~dB}$ in a broad bandwidth of $20 \mathrm{~nm}^{12}$. Average insertion loss was $0.18 \pm 0.03 \mathrm{~dB}$ and crosstalk was $-41 \pm 2 \mathrm{~dB}^{13}$. The waveguide crossing only operated at $1550 \mathrm{~nm}$ and $1310 \mathrm{~nm}$, the results showed transmission insertion loss of $-0.028 \pm 0.009 \mathrm{~dB}$ only for the $1550 \mathrm{~nm}$ device and $-0.017 \pm 0.005 \mathrm{~dB}$ only for the $1310 \mathrm{~nm}$ device. Both crossings show crosstalk lower than $-37 \mathrm{~dB}^{14}$. Another waveguide crossing showed an insertion loss of $1.82 \mathrm{~dB}$ as well as a crosstalk of $<-18 \mathrm{~dB}$ from 1,510 to $1600 \mathrm{~nm}^{15}$. However, the footprint of the device was as compact as $21 \times 21 \mu \mathrm{m}^{2}$. A dual-mode waveguide crossing is proposed. The characterization results for the fabricated device show that low insertion loss below $1.5 \mathrm{~dB}$ and low crosstalk below $-18 \mathrm{~dB}$ can be achieved ${ }^{16}$. But, it must need two waveguide crossing to achieve the function. Han et al. ${ }^{17}$ used particle swarm optimization algorithm to design a SOI waveguide crossing with a footprint of only $\sim 1 \times 1 \mu \mathrm{m}^{2}$. Although it has an insertion loss $<0.175 \mathrm{~dB}$ and crosstalk around $-30 \mathrm{~dB}$ for the C-band, the designed method needs complex calculations and long-time simulation, and the number of iterations is set 500 . A waveguide cross was designed that insertion loss and crosstalk were less than $0.6 \mathrm{~dB}$ and $-24 \mathrm{~dB}$ from 1530 to $1590 \mathrm{~nm}$, respectively ${ }^{18}$. However, the fabricated device still occupied a footprint of $4.8 \times 4.8 \mu \mathrm{m}^{2}$. Although traditional methods have been used to design devices with good performance, the footprint is always large that has a negative impact on dense integration, and the design of such devices rely heavily on the intuition and experience of the designer and cannot manually achieve a full-parameter space design. Therefore, we should use other methods to design waveguide bends and crossings. This method must be simple and feasible. It should greatly reduce the footprint of the device and achieve superior high performance.

In recent years, the inverse design method and DBS algorithm of ultra-compact silicon photonic devices have drawn more and more attentions ${ }^{19-25}$. The best-known institutions for inverse design research are the University of Utah and Stanford. The University of Utah achieved the inverse design of integrated nano-photonic polarization beam splitters by combining DBS algorithm and the current commercial software ${ }^{19}$. The Stanford used alternating directions method of multipliers (ADMM) to design a wavelength demultiplexer of $1300 \mathrm{~nm} / 1500 \mathrm{~nm}^{20}$. For other optical device designs like flat optics and holograms, the inverse design also has a wide range of application ${ }^{26-29}$. Learning from their train of thought, Harbin Institute of Technology ${ }^{30-32}$, Huazhong University of Science and Technology ${ }^{33-35}$, National University of Defense Technology ${ }^{36-40}$ designed a variety of on-chip optical devices and achieved a good performance. One of the ways to realize inverse design is combining DBS algorithm and commercially available simulation software capable of implementing FDTD solutions, such as Lumerical FDTD Solutions ${ }^{41}$. Inspired by them, we used inverse design and DBS algorithm to design waveguide bends and crossings.

In this paper, based on SOI platform, we successfully demonstrated a 90-degree waveguide bend, a waveguide crossing and a same direction waveguide crossing in the entire wavelength range of 1,400-1600 nm by using DBS algorithm. Their footprints are only $2.4 \times 2.4 \mu \mathrm{m}^{2}, 2.4 \times 2.4 \mu \mathrm{m}^{2}, 2.4 \times 3.6 \mu \mathrm{m}^{2}$ respectively. The transmission efficiency of the 90 -degree waveguide is $>0.5 \mathrm{~dB}$ in the entire wavelength range of $1,440-1640 \mathrm{~nm}$ and up to $0.18 \mathrm{~dB}$ at some wavelengths. The waveguide crossing can provide insertion loss of less than $0.5 \mathrm{~dB}$. The same direction waveguide crossing can provide the insertion loss of less than $0.56 \mathrm{~dB}$. The measured crosstalk of the two crossings is lower than $-19 \mathrm{~dB}$ and $-21 \mathrm{~dB}$ respectively. Meanwhile, four kinds of optical interconnection modes are designed with the proposed bending and crossing, which realize the superior performance. Compared with traditional optical interconnection, the integration density and benefit various on-chip optical systems are greatly improved.

\section{Results}

Optical interconnection component. A 90-degree waveguide bend. Based on the "design method", we first design a 90-degree waveguide bend on a silicon-on-insulator (SOI) substrate with $220 \mathrm{~nm}$ silicon on top of $3 \mu \mathrm{m}$ buried oxide. As shown in Fig. $1 \mathrm{a}-\mathrm{c}$, the computation domain is $2.4 \times 2.4 \mu \mathrm{m}^{2}$, where the optimization region is divided into $20 \times 20$ pixels and each pixel is a square of $120 \mathrm{~nm} \times 120 \mathrm{~nm}$ with a central circular hole. The hole has a diameter of $90 \mathrm{~nm}$ and a depth of $220 \mathrm{~nm}$, which can be fabricated with an electron-beam lithography (EBL) system (Vistec EBPG 5000 Plus).

Each pixel can occupy two states: silicon or air. The white and red regions of the pixel denote the etched area where no silicon remains on top of the oxide and the unetched area where the $220 \mathrm{~nm}$ silicon remains, respectively. At the beginning, all the pixel states are chosen to be silicon, and the excitation the $\mathrm{TE}_{0}$ mode in the wavelength range of 1,400-1600 $\mathrm{nm}$. Then the state of each pixel is altered one by one, and the figure-of-merit (FOM) is inspected. Here the FOM is defined as:

$$
\mathrm{FOM}=\mathrm{T}_{\text {out_port }}
$$

where $\mathrm{T}_{\text {out_port }}$ is the transmission efficiency of the final structure at the wavelength of $1550 \mathrm{~nm}$ as the input light source is $\mathrm{TE}_{0}$ mode, which is calculated by $3 \mathrm{D}$ FDTD with $40 \mathrm{~nm} \times 40 \mathrm{~nm} \times 40 \mathrm{~nm}$ grid size and 8 layers PML. If the FOM is improved, the pixel state is retained. If not, the pixel state is reversed and the algorithm proceeds to the next pixel. One iteration ends after all the pixel states are inspected. Then the iterations continue until the FOM does not improve further.

We perform the device design and optimization using an eight-core desktop. For the 90-degree waveguide bend, it takes $\sim 24 \mathrm{~h}$ on average to get the final results after 4 iterations. Given that fabrication variations can have a significant impact on real-world device performance, we characterize the same bends with $\pm \pm 10 \%$ variation in pillar diameter by simulation. The simulated optical field profile of the device when the continuous wave is launched from input port is shown in Fig. 1d. Figure 1e shows the calculated normalized transmission efficiency. The simulated normalized transmission efficiency of the bend is $<0.5 \mathrm{~dB}$ in the entire range of $1,400-1600 \mathrm{~nm}$ and up to $0.18 \mathrm{~dB}$ at some wavelength. As we can show, a $\pm 10 \%$ variation in pillar diameter doesn't affect largely on the 90 -degree waveguide bend. It is seen from the Table 1 that the waveguide bend we designed has very low loss losses and a relatively small footprint compared with other waveguide bends. The method we used is feasible and auto-actuated. 
(a)

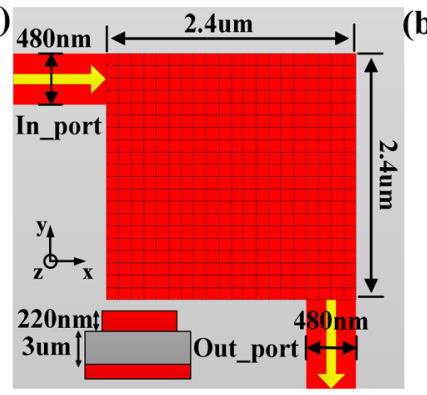

(b)

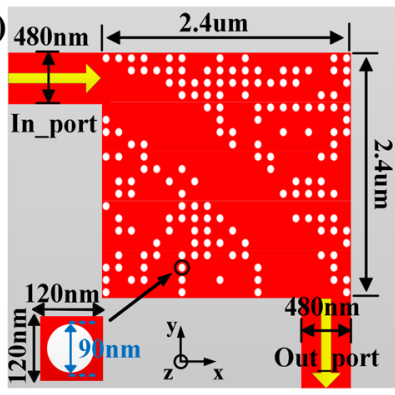

(c)

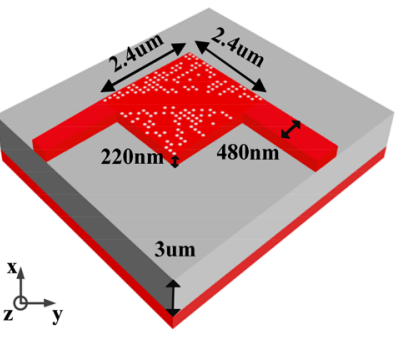

Silicon

(d)

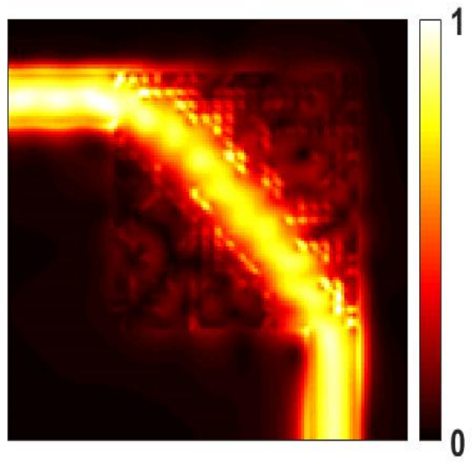

(e)

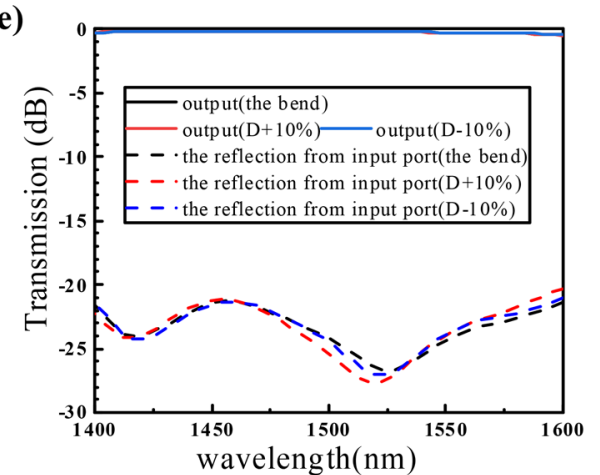

Figure 1. Design and simulation results of the 90-degree waveguide bend. (a) Initial silicon slab before design optimization. (b) Final optimized structure. (c) Isometric view. (d) Simulated optical field distributions. (e) Simulated normalized transmission spectra. Here, we have plotted the electromagnetic energy density $U=\epsilon|\mathbf{E}|^{2}+\mu|\mathbf{H}|^{2}$. An animated version of $\mathbf{d}$ is available in Supplementary Video S1.

\begin{tabular}{|l|l|l|l|l|l|}
\hline References & Bending area $\left(\boldsymbol{\mu m}^{2}\right)$ & Propagation losses $(\mathbf{d B})$ & Waveguide & Method & Note \\
\hline This work & $2.4 \times 2.4$ & 0.18 & Etched silicon & DBS & Auto-actuated \\
\hline 2 & $5 \times 5$ & 0.023 & $\sim 10 \mu \mathrm{m}$ long waveguide & Variational optimization & Hand-actuated \\
\hline${ }^{3}$ & $50 \times 50$ & 0.2 & $\sim 100 \mu \mathrm{m}$ long waveguide & Numerical analysis & Hand-actuated \\
\hline${ }^{4}$ & $275 \times 275$ & $\sim 0.1$ & $\sim 550 \mu \mathrm{m}$ long waveguide & Beam-propagation & Hand-actuated \\
\hline 5 & $5 \times 5$ & 0.027 & $25 \mu \mathrm{m}$ long waveguide & Manual adjustment & Hand-actuated \\
\hline 6 & $2 \times 2$ & 0.086 & $21 \mathrm{~mm}$ long waveguide & Manual adjustment & Hand-actuated \\
\hline 7 & $4 \times 4$ & 0.5 & $\sim 8 \mu \mathrm{m}$ long waveguide & Clothoid curves & Hand-actuated \\
\hline 8 & $10 \times 10$ & 0.02 & $\sim 20 \mu \mathrm{m}$ long waveguide & Micron-scale cores & Hand-actuated \\
\hline
\end{tabular}

Table 1. Comparison of the waveguide bends.

\section{Waveguide crossing.}

- A waveguide crossing

The on-chip optical interconnection inevitably needs to address the waveguide cross-connect problem. Herein, we design a waveguide crossing with a footprint of only $2.4 \times 2.4 \mu \mathrm{m}^{2}$ using the same process as the bend, which has a quadrature symmetry. The structural schematic diagram of the device is described in Fig. 2a,b, the crossing has two input ports labeled as I1 and I2, and two output ports labeled as $\mathrm{O} 1-\mathrm{O} 2$, respectively. The widths of the two input waveguides and the output waveguide are $480 \mathrm{~nm}$.

This design region is also divided into $20 \times 20$ discrete pixels with minimum feature size of $90 \mathrm{~nm}$. In the optimizing process, we use the $\mathrm{TE}_{0}$ mode in the wavelength range of $1,400-1600 \mathrm{~nm}$ as the only one light source placed at I1 port. The FOM of the crossing for inverse design is defined as:

$$
\mathrm{FOM}=\left[\mathrm{T}_{1}(\mathrm{i}, \mathrm{j}+1)>\mathrm{T}_{1}(\mathrm{i}, \mathrm{j})\right] \cap\left[\mathrm{T}_{2}(\mathrm{i}, \mathrm{j}+1) \leq \mathrm{T}_{2}(\mathrm{i}, \mathrm{j})\right]
$$

where $T_{1}(i, j)$ and $T_{1}(i, j+1)$ represent the average transmission of $O 1$ port in the ith iterate of $j$ th and $(j+1)$ th pixel, respectively. $\mathrm{T}_{2}(\mathrm{i}, \mathrm{j})$ and $\mathrm{T}_{2}(\mathrm{i}, \mathrm{j}+1)$ represent the average transmission of $\mathrm{O} 2$ port in the ith iterate of $j$ th and 
(a)

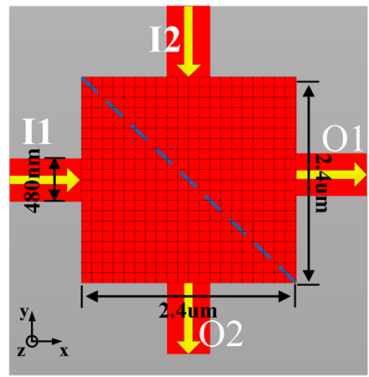

(b)

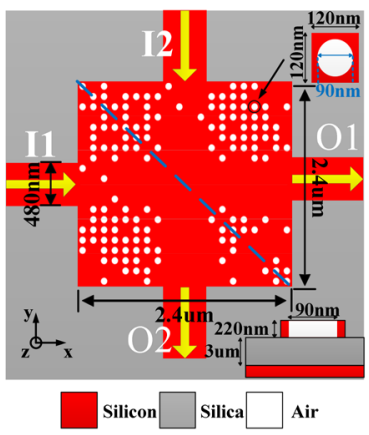

(c)

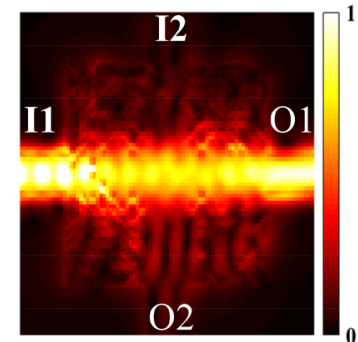

(e)

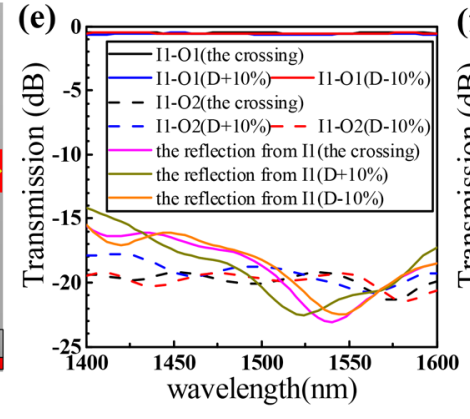

(d)

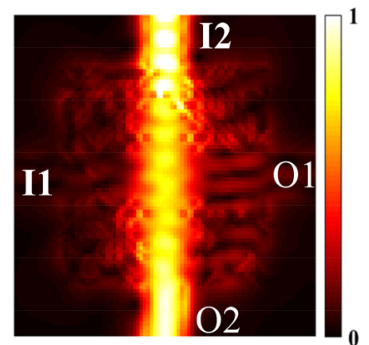

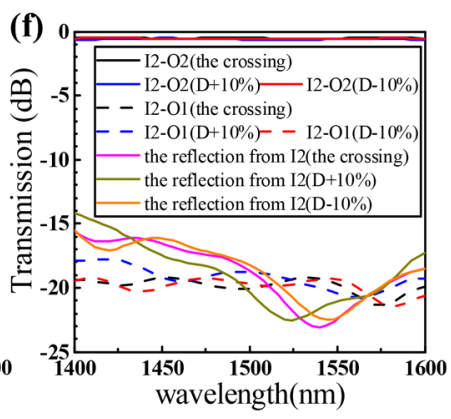

Figure 2. Design and simulation results of the waveguide crossing. (a) Initial silicon slab before design optimization. (b) Final optimized structure. (c), (d) Simulated optical field distributions. (e), (f) Simulated normalized transmission spectra. Here, we have plotted the electromagnetic energy density $U=\epsilon|\mathbf{E}|^{2}+\mu|\mathbf{H}|^{2}$. An animated version of $(\mathbf{c})$ is available in Supplementary Video S2.

$(\mathrm{j}+1)$ th pixel, respectively. All the transmissions are calculated by 3D FDTD with $40 \mathrm{~nm} \times 40 \mathrm{~nm} \times 40 \mathrm{~nm}$ grid size and 8 layers PML. Actually, the first term in the right of Eq. (2) is used to optimize average insertion loss (IL) and the second one is employed to minimize the crosstalk (CT). If FOM=1, it means IL is improved and $\mathrm{CT}$ descend, the pixel state is retained. If not, the pixel state is reversed.

It takes about $36 \mathrm{~h}$ after 4 iterations to get the optimized crossing on an eight-core desktop. To investigate the manufacturing error of the crossing, we test the same crossing with a $\pm 10 \%$ variation in pillar diameter. Figure 2c,d shows the simulated optical. The simulated ILs and CTs spectra at O1and O2 ports are plotted in Fig. 2e,f when the continuous wave is launched from input ports I1 and I2, respectively. The simulated CTs for the crossing and the same crossings with $\pm 10 \%$ diameter are $<-19 \mathrm{~dB},<-18 \mathrm{~dB},<-19 \mathrm{~dB}$, respectively. The simulated ILs for all devices are $\sim 0.5 \mathrm{~dB}$ from 1,400 to $1,600 \mathrm{~nm}$ wavelength range. $\mathrm{A} \pm 10 \%$ variation in pillar diameter had nearly negligible impact on the IL. Due to a symmetric structure, the results obtained as the light source placed at I1 and I2 ports are consistent.

\section{- A same direction waveguide crossing}

Here, we design another same direction waveguide crossing. As shown in Fig. 3a,b, the inverse design region composed of $20 \times 30$ discrete pixels occupies a compact footprint of only $2.4 \times 3.6 \mu \mathrm{m}^{2}$. The crossing is optimized using the same process as the waveguide crossing. The FOM is also defined as Eq. (2). It takes about $48 \mathrm{~h}$ after 5 iterations to get the final optimized structure on an eight-core desktop. Figure $3 \mathrm{c}$,d shows the simulated optical and Fig. 3e,f shows the calculated ILs and CTs spectra when the continuous wave is launched from I1 and I2 ports, respectively. For the final crossing, the average IL is $\sim 0.56 \mathrm{~dB}$ and the $\mathrm{CT}$ is $<-21 \mathrm{~dB}$ within the wavelength range from 1,400-1600 $\mathrm{nm}$. Even though the diameter of the crossing changes with a $\pm 10 \%$ variation, the IL and CT curves are still quite consistent. Table 2 summarizes results from some existing literature. It is seen that the waveguide crossings we designed have a small footprint. In contrast with other waveguide crossings, the measured insertion loss and crosstalk are relatively low.

Optical interconnection integrated structures. In principle, the optical signal can be delivered to any chip locations via any routing path provided the low loss bending and crossing are available. For a proof-of-concept demonstration, we arbitrarily design four optical interconnection integrated structures with the proposed waveguide bend and crossings.

The first structure consists of four waveguide crossings, which is named “2_2_2_2" structure. The schematic diagram of this structure is shown in Fig. $4 \mathrm{a}$, its footprint is $2.4 \times 5.3 \mu \mathrm{m}^{2}$, the span between the four crossings is $500 \mathrm{~nm}$, and the input waveguides and the output waveguide are $480 \mathrm{~nm}$. We define input ports I1-I4 and output ports $\mathrm{O} 1-\mathrm{O} 4$, respectively. Figure $4 \mathrm{~b}-\mathrm{e}$ shows the simulated optical field when the continuous wave is launched from I1-I4 ports, respectively. The match IL and CT spectra are plotted in Fig. 4f-i. The result curves in Fig. 4f,h, and $g, i$ are consistent due to the symmetric structure. The average $\mathrm{IL}$ is $\sim 1 \mathrm{~dB}$ and the CTs are $<-18 \mathrm{~dB}$ within the wavelength range from 1,400 to $1600 \mathrm{~nm}$. 
(a)

(b)
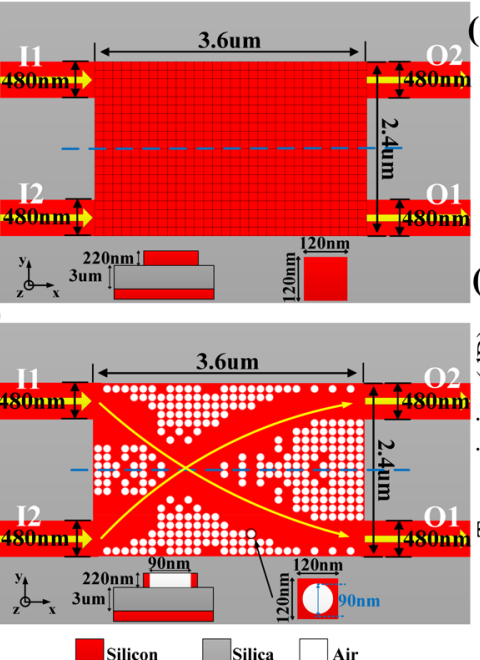

(c)

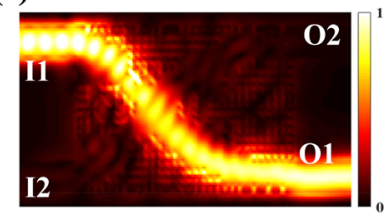

(d)

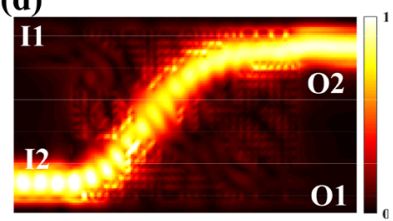

(e)

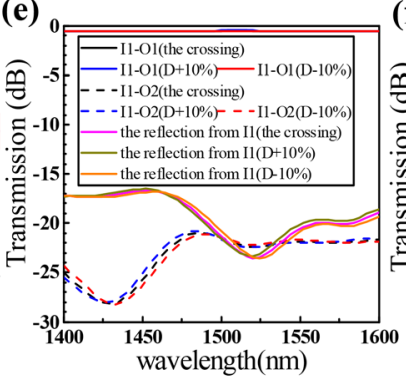

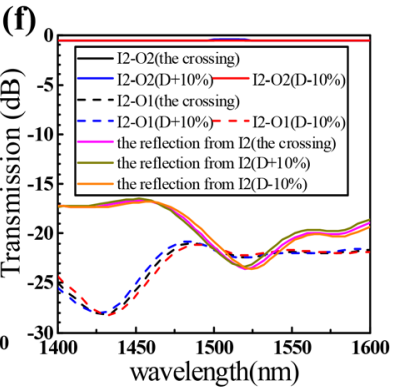

Figure 3. Design and simulation results of the same direction waveguide crossing. (a) Initial silicon slab before design optimization. (b) Final optimized structure. (c), (d) Simulated optical field distributions. (e), (f) Simulated normalized transmission spectra. Here, we have plotted the electromagnetic energy density $U=\epsilon|\mathbf{E}|^{2}+\mu|\mathbf{H}|^{2}$. An animated version of $(\mathbf{c})$ is available in Supplementary Video S3.

\begin{tabular}{|l|l|l|l|l|l|}
\hline References & Footprint $\left(\boldsymbol{\mu m}^{2}\right)$ & IL $(\mathbf{d B})$ & CT $(\mathbf{d B})$ & Method & Note \\
\hline \multirow{2}{*}{ This work } & $2.4 \times 2.4$ & 0.5 & -19 & DBS & Auto-actuated \\
\cline { 2 - 5 } & $2.4 \times 3.6$ & 0.56 & -21 & DBS & Auto-actuated \\
\hline 9 & $175.2 \times 175.2$ & 0.009 & $<-37$ & Self-imaging & Large footprint \\
\hline 10 & $13 \times 13$ & 0.4 & -30 & Manual adjustment & Multiple experiments \\
\hline 11 & $6 \times 6$ & 1.7 & -40 & Parabolic taper & Hand-actuated \\
\hline 12 & $6 \times 6$ & 0.2 & -40 & GA & 20 nm bandwidth \\
\hline 13 & $6 \times 6$ & 0.18 & -41 & PSO & 50 generations \\
\hline 14 & $6 \times 6$ & $0.028,0.017$ & -37 & Step by step & Only operating at $1550 \mathrm{~nm}$ and $1310 \mathrm{~nm}$ \\
\hline 15 & $21 \times 21$ & 1.82 & -18 & Y-junction & Large footprint \\
\hline 16 & $2.4 \times 2.4$ & 1.5 & -18 & Self-imaging & Requiring two crossings \\
\hline 17 & $1 \times 1$ & 0.175 & -30 & PSO & 500 generations \\
\hline 18 & $4.8 \times 4.8$ & 0.6 & -24 & Symmetric & Still large \\
\hline
\end{tabular}

Table 2. Comparison of the waveguide crossings.

(a)

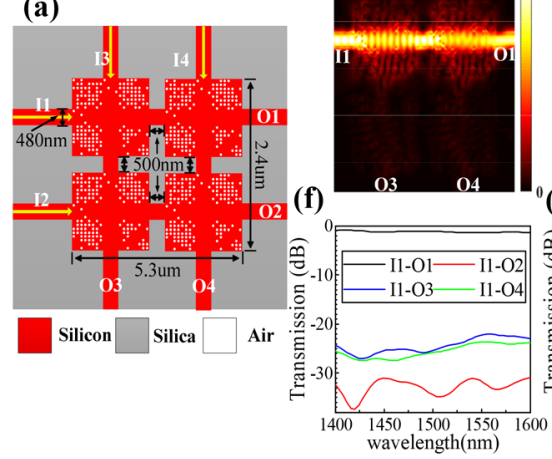

(c)

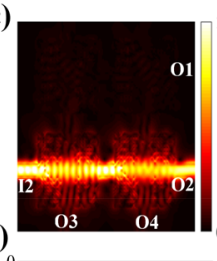

(g)

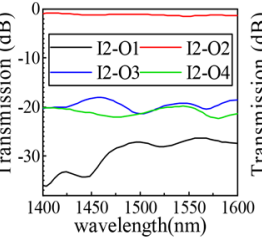

(d)

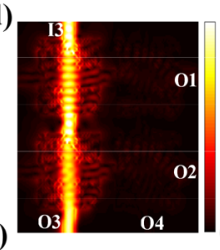

(h)

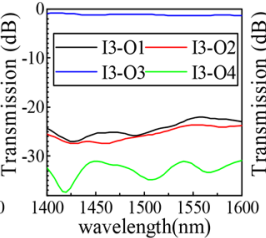

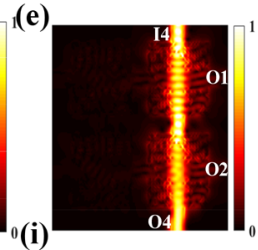

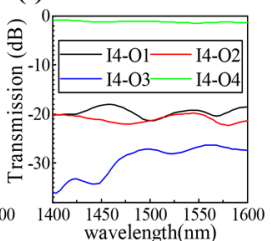

Figure 4. Design and simulation results of the "2_2_2_2" structure. (a) Schematic diagram of the structure. (b)-(e) Simulated optical field distributions. (f)-(i) Simulated normalized transmission spectra. 
(a)

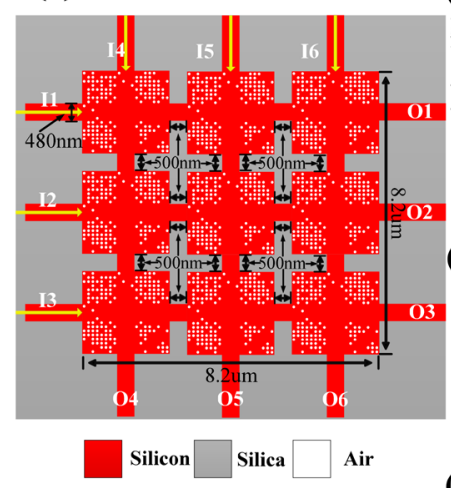

(b)

(e)

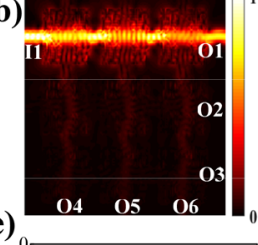

兽

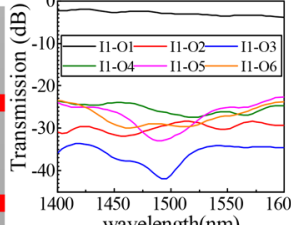

(h)

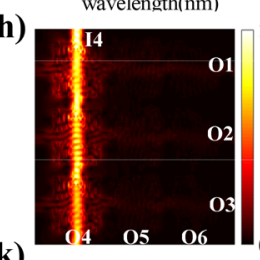

(k)

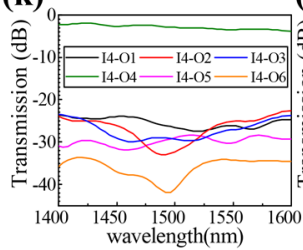

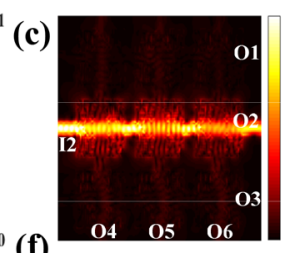

(f)

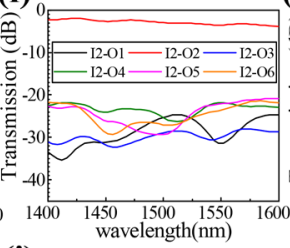

(i)

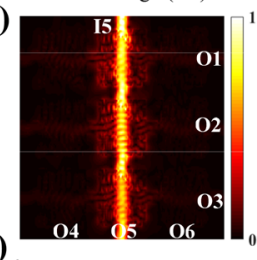

(I)

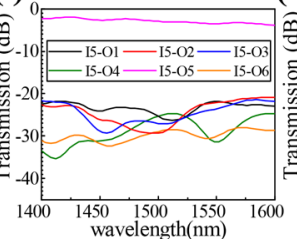

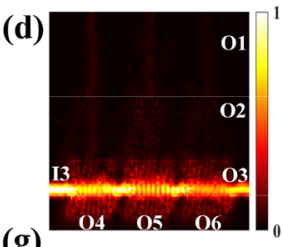

(g)
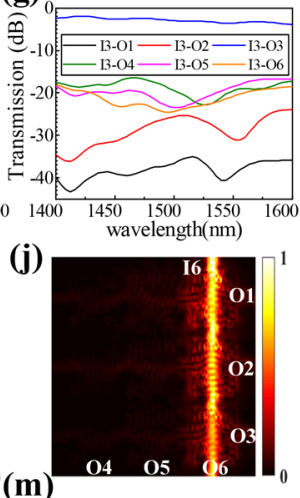

$(\mathbf{m})$

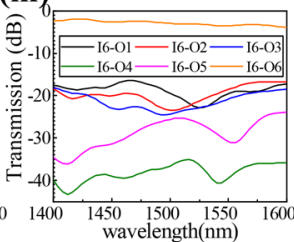

Figure 5. Design and simulation results of the "3_3_3_3" structure. (a) Schematic diagram of the structure. (b)-(d) (i)-(k) Simulated optical field distributions. (e)-(g) (l)-(n) Simulated normalized transmission spectra.

The second structure with a footprint of $8.2 \times 8.2 \mu \mathrm{m}^{2}$ consists of nine waveguide crossings, which is named "3_3_3_3" structure. The relevant specific parameters of this structure are shown in Fig. 5a. We define input ports I1-I6 and output ports $\mathrm{O} 1-\mathrm{O} 6$, respectively. Figure $5 \mathrm{~b}-\mathrm{d}$ and $\mathrm{h}-\mathrm{j}$ show the simulated optical field when the continuous wave is launched from I1-I6 ports, respectively. The match IL and CT spectra are plotted in Fig. 5e-g and $\mathrm{k}-\mathrm{m}$. The result curves in Fig. $5 \mathrm{e}$ and $\mathrm{k}, \mathrm{f}$ and $\mathrm{l}, \mathrm{g}$ and $\mathrm{m}$ are consistent due to the symmetric structure. The average IL for all output ports is $2.5 \mathrm{~dB}$ from 1,400 to $1,600 \mathrm{~nm}$. The CTs are measured to be $<-16 \mathrm{~dB}$ within the same wavelength range.

The third structure with a footprint of $7.7 \times 6.14 \mu \mathrm{m}^{2}$ consists of three same direction waveguide crossings, which is named "4_4" structure. The labels of input ports and output ports and other relevant specific parameters of this structure are shown in Fig. 6a. Figure $6 \mathrm{~b}-\mathrm{e}$ shows the simulated optical field when the continuous wave is launched from I1-I4 ports, respectively. The match IL and CT spectra are plotted in Fig. $6 \mathrm{f}-\mathrm{i}$. The result curves in Fig. $6 \mathrm{f}$ and $\mathrm{i}, \mathrm{g}$ and $\mathrm{h}$ are consistent due to the symmetric structure. When the input waves from I1 or I4 input ports, the losses come from the one times cross connection, and the average IL is $\sim 0.6 \mathrm{~dB}$ and the CTs are $<-20 \mathrm{~dB}$ within the wavelength range from 1,400 to $1600 \mathrm{~nm}$. When the input waves from I2 or I3 input ports, the losses come from the two times cross connection, and the average IL is $\sim 1.2 \mathrm{~dB}$ from 1,400 to $1,600 \mathrm{~nm}$, the CTs are measured to be $<-16 \mathrm{~dB}$ within the same wavelength range.

The fourth structure with a footprint of $10.12 \times 10.12 \mu \mathrm{m}^{2}$ consists of three waveguide bends and three waveguide crossing, which is named " $3 \_3$ " structure. The relevant specific parameters of this structure are shown in Fig. 7a. We define input ports I1-I3 and output ports O1-O3, respectively. Figure $7 \mathrm{~b}-\mathrm{d}$ shows the simulated optical field when the continuous wave is launched from I1-I3 ports, respectively. The match IL and CT spectra are plotted in Fig. 7e-g. The average ILs for $\mathrm{O} 1, \mathrm{O} 2$ and $\mathrm{O} 3$ output ports are $\sim 2.5 \mathrm{~dB}, \sim 2 \mathrm{~dB}$ and $\sim 3 \mathrm{~dB}$ from 1,500 to $1,580 \mathrm{~nm}$, respectively. The CTs are for $\mathrm{O} 1, \mathrm{O} 2$ and $\mathrm{O} 3$ output ports measured to be $<-27 \mathrm{~dB},<-25 \mathrm{~dB}$ and $<-21 \mathrm{~dB}$ within the same wavelength range, respectively.

The above is a few examples of optical interconnect structures using the waveguide bend and crossings. It is obviously that the number of devices contributes to the losses of the structures. The more devices we use, the greater the losses. The minimum intervals between all devices is $500 \mathrm{~nm}$ making sure there is not any out-ofplane light coupling.

\section{Discussion}

In this paper, we use inverse design concept and DBS algorithm to design ultra-compact high efficiency and low crosstalk waveguide bend and crossings for optical interconnection based on SOI platform. The footprints of them are only $2.4 \times 2.4 \mu \mathrm{m}^{2}, 2.4 \times 2.4 \mu \mathrm{m}^{2}$ and $2.4 \times 3.6 \mu \mathrm{m}^{2}$ respectively. The waveguide bend realizes the 90 degree bending of light, up to $0.18 \mathrm{~dB}$ transmission efficiency. The ILs and CTs of the waveguide crossings 
(a)

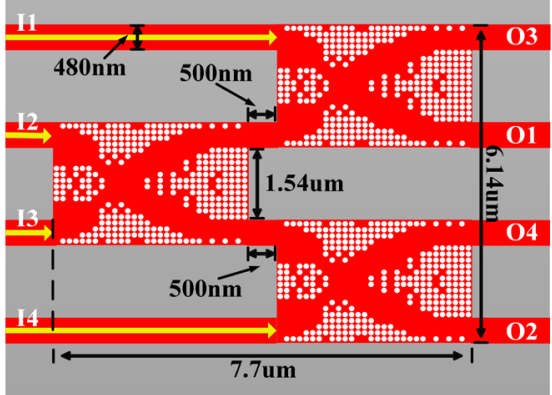

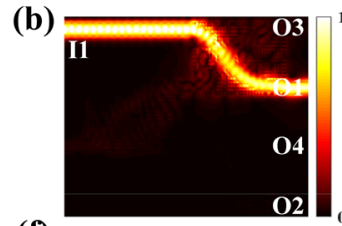

(f)

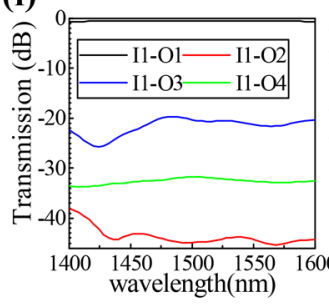

(g)
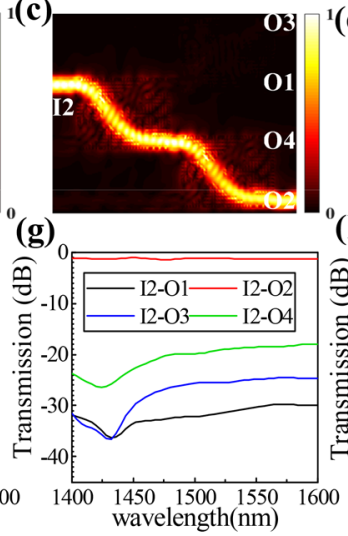

Silicon

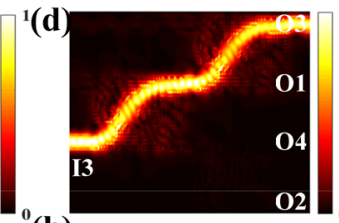

(h)

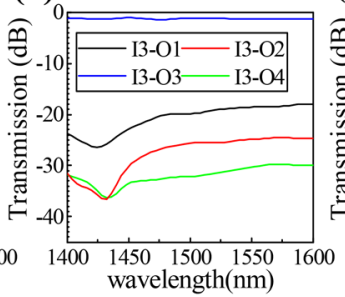

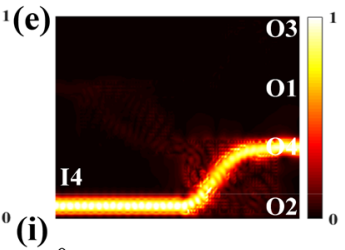

(i)

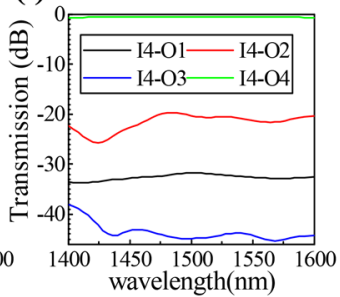

Figure 6. Design and simulation results of the " $4 \_4$ " structure. (a) Schematic diagram of the structure. (b)-(e) Simulated optical field distributions. (f)-(i) Simulated normalized transmission spectra.

(a)

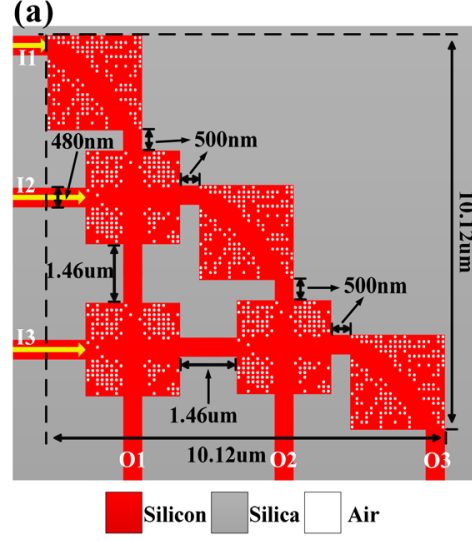

(b)

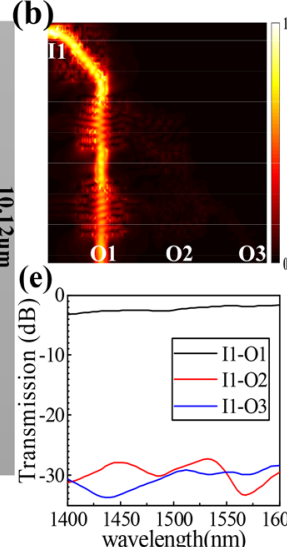

(c)

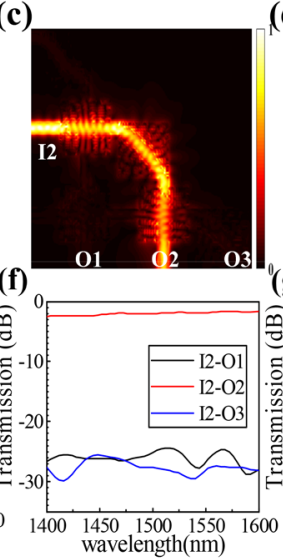

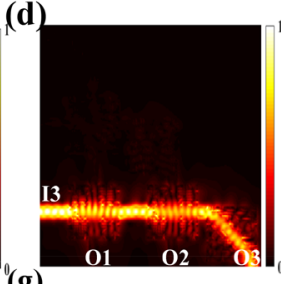

$(\mathrm{g})$

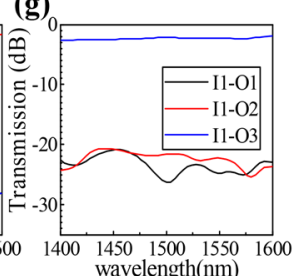

Figure 7. Design and simulation results of the " $3 \_3$ " structure. (a) Schematic diagram of the structure. (b)-(d) Simulated optical field distributions. (e)-(g) Simulated normalized transmission spectra.

are less than $0.5 \mathrm{~dB}, 0.56 \mathrm{~dB},-19 \mathrm{~dB}$ and $-21 \mathrm{~dB}$, respectively. Furthermore, we arbitrarily design four optical interconnection integrated structures with the proposed waveguide bend and crossings. All of them exhibit high performances. Actually, more optical interconnect structures can be achieved to deliver optical signal using them. The ultra-compact structures will significantly improve the integration density and benefit various on-chip optical systems. Meantime, our design thought and algorithm flow can also be applied to the research and design of other on-chip photonic devices, such as the optical power beam splitter, the mode division multiplexer, the wavelength division multiplexer, etc.

Design method. The overall design idea of inverse design: Firstly, setting the target performance of the devices. Then, according to the set performance requirements, the devices are designed and optimized by using 
various optimization algorithms. Here, we use DBS algorithm to design the devices. The general process is as follows:

(1) Dividing pixels: The optimized region is divided into a certain number of pixels. The geometry of each pixel can be circle, square, or other complex shapes. The minimum feature size can be determined according to the fabrication capability. In this paper, each pixel is a square of $120 \mathrm{~nm} \times 120 \mathrm{~nm}$ with a central circular hole. The hole has a diameter of $90 \mathrm{~nm}$.

(2) Establishing the initial pixel states: Each pixel can occupy two states: silicon or air. For the initial pattern of the devices, all the pixel states are chosen to be silicon in this paper.

(3) Defining figure-of-merit (FOM): the FOM is used to see if the pixel state is witched. Different FOMs are defined according to different devices. In this paper, the FOM is defined as Eqs. (1) or (2).

(4) Witching the pixel state: the state of each pixel is altered one by one, and the FOM is inspected. The pixel state is retained if the FOM is improved. If not, the pixel state is reversed and the algorithm proceeds to the next pixel. One iteration ends after all the pixel states are inspected.

(5) Iterative refinement: The iterations continue until the FOM does not improve further.

\section{Data availability}

All data generated or analyzed during this study are included in this article.

Received: 24 February 2020; Accepted: 29 June 2020

Published online: 20 July 2020

\section{References}

1. Miller, S. E. Integrated optics: an introduction. Bell Labs Tech. J. 48(7), 2059-2069 (1969).

2. Bahadori, M., Bahadori, M., Cheng, Q. \& Bergman, K. Universal design of waveguide bends in silicon-on-insulator photonics platform. J. Lightw. Technol. 37(13), 3044-3054 (2019).

3. Vogelbacher, F. et al. Analysis of silicon nitride partial Euler waveguide bends. Opt. Exp. 27(22), 31394-31406 (2019).

4. Hu, Z. \& Lu, Y. Computing optimal waveguide bends with constant width. J. Lightw. Technol. 25(10), 3161-3167 (2007).

5. Bogaerts, W. \& Selvaraja, S. K. Compact single-mode silicon hybrid rib/strip waveguide with adiabatic bends. IEEE Photon. J. 3(3), 422-432 (2011).

6. Vlasov, Y. A. \& McNab, S. J. Losses in single-mode silicon-on-insulator strip waveguides and bends. Opt. Exp. 12(8), 1622 (2004).

7. Fujisawa, T., Makino, S., Sato, T. \& Saitoh, K. Low-loss, compact, and fabrication-tolerant Si-wire $90^{\circ}$ waveguide bend using clothoid and normal curves for large scale photonic integrated circuits. Opt. Exp. 25(8), 9150-9159 (2017).

8. Cherchi, M., Ylinen, S., Harjanne, M., Kapulainen, M. \& Aalto, T. Dramatic size reduction of waveguide bends on a micron-scale silicon photonic platform. Opt. Exp. 21(15), 17814 (2013).

9. Liu, H., Tam, H., Wai, P. \& Pun, E. Low-loss waveguide crossing using a multimode interference structure. Opt. Commun. 241(1), 99-104 (2004).

10. Chen, H. \& Poon, A. W. Low-loss multimode-interference-based crossings for silicon wire waveguides. IEEE Photon. Technol. Lett. 18(21), 2260-2262 (2006).

11. Bogaerts, W., Dumon, P., Van, T. D. \& Baets, R. Low-loss, low-crossing-talk crossings for silicon-oninsulator nanophotonic waveguides. Opt. Lett. 32(19), 2801-2803 (2007).

12. Sanchis, P. et al. Highly efficient crossing structure for silicon-on-insulator waveguides. Opt. Lett. 34(18), 2760-2762 (2009).

13. Zhang, Y. et al. A CMOS-compatible, low-loss, and low-crossingtalk silicon waveguide crossing. IEEE Photon. Technol. Lett. 25(5), 422-425 (2013).

14. Ma, Y. et al. Ultralow loss single layer submicron silicon waveguide crossing for SOI optical interconnect. Opt. Exp. 21(24), 29374-29382 (2013).

15. Sun, C., Yu, Y. \& Zhang, X. Ultra-compact waveguide crossing for a mode-division multiplexing optical network. Opt. Lett. 42(23), 4913-4916 (2017).

16. Xu, H. \& Shi, Y. Dual-mode waveguide crossing utilizing taper-assisted multimode-interference couplers. Opt. Lett. 41(23), 53815384 (2016).

17. Han, H. et al. High performance ultra-compact SOI waveguide crossing. Opt. Exp. 26(20), 25602-25610 (2018).

18. Chang, W. et al. Ultracompact dual-mode waveguide crossing based on subwavelength multimode-interference couplers. Photon. Res. 6(20), 660-665 (2018).

19. Shen, B., Wang, P., Polson, R. \& Menon, R. An integrated-nanophotonics polarization beamsplitter with $2.4 \times 2.4 \mu \mathrm{m}^{2}$ footprint. Nat. Photon. 9(6), 378 (2015).

20. Lu, J. \& Vučković, J. Nanophotonic computational design. Opt. Exp. 21(11), 13351-13367 (2013).

21. Meem, M. et al. Broadband lightweight flat lenses for long-wave infrared imaging. PNAS 43, 21375-21378 (2019).

22. Meem, M., Meem, A. \& Menon, R. Multi-plane, multi-band image projection via broadband diffractive optics. Appl. Opt. 59(1), 38-44 (2019).

23. Xiao, T. et al. Diffractive spectral-splitting optical element designed by adjoint-based electromagnetic optimization and fabricated by femtosecond 3D direct laser writing. ACS Photon. 3(5), 886-894 (2016).

24. Su, L., Piggott, A. Y., Sapra, N. V., Petykiewicz, J. \& Vuckovic, J. Inverse design and demonstration of a compact on-chip narrowband three-channel wavelength demultiplexer. ACS Photon. 5(2), 301-305 (2018).

25. Sapra, N. V. et al. Inverse design and demonstration of broadband grating couplers. IEEE J. Sel. Top. Quantum Electron. 25(3), 1-7 (2019).

26. Shen, B., Polson, R. \& Menon, R. Metamaterial-waveguide bends with effective bend radius < $\lambda 0 / 2$. Opt. Lett. 40(24), 5750-5753 (2015).

27. Majumder, A., Shen, B., Shen, R. \& Menon, R. Ultra-compact polarization rotation in integrated silicon photonics using digital metamaterials. Opt. Exp. 25(17), 19721-19731 (2017).

28. Tahersima, M. H. et al. Deep neural network inverse design of integrated photonic power splitters. Sci. Rep. 9, 1368 (2019).

29. Turduev, M. et al. Ultracompact photonic structure design for strong light confinement and coupling into nanowaveguide. J. Lightw. Technol. 36(14), 2812-2819 (2018).

30. Xu, K. et al. Integrated photonic power divider with arbitrary power ratios. Opt. Lett. 42(4), 855-858 (2017).

31. Xie, H. et al. Inversely designed $1 \times 4$ power splitter with arbitrary ratios at 2- $\mu \mathrm{m}$ spectral band. IEEE Photon. J. 10(4), 1-6 (2018).

32. Liu, Y. et al. Arbitrarily routed mode-division multiplexed photonic circuits for dense integration. Nat. Commun. 10(1), 1-7 (2019). 
33. Lu, L., Liu, D., Yan, M. \& Zhang, M. Subwavelength adiabatic multimode Y-junctions. Opt. Lett. 44(19), 4729-4732 (2019).

34. Chang, W. et al. An ultra-compact multimode waveguide crossing based on subwavelength asymmetric Y-junction. IEEE Photon. J. 10, 1-8 (2018).

35. Chang, W. et al. Ultra-compact mode (de)multiplexer based on subwavelength asymmetric Y-junction. Opt. Exp. 26(7), 8162-8170 (2018).

36. Huang, J. et al. Ultra-compact broadband polarization beam splitter with strong expansibility. Photon. Res. 6(6), 574-578 (2018).

37. Huang, J. et al. Implementation of on-chip multi-channel focusing wavelength demultiplexer with regularized digital metamaterials. Nanophotonics. 9(1), 159-166 (2019).

38. Li, Z., Zhang, Z., Huang, J. \& Yang, J. genetically designed ultra-compact wideband multiple-use reflectors with high reflectivity appling to optics communication. ICCT(2019). 724-728. https://doi.org/10.1109/ICCT46805.2019.8947154 (2019).

39. Han, J., et al. Inverse designed ultra-compact dual-channel wavelength demultiplexer with plasmonic subwavelength structure. ACPC(2019). Paper M4A. 283 (2019).

40. Ma, H., Huang, J., Zhang, K. \& Yang, J. Arbitrary-direction, multichannel and ultra-compact power splitters by inverse design method. Opt. Commun. 462, 125329. https://doi.org/10.1016/j.optcom.2020.125329 (2020).

41. Lumerical FDTD Solutions. https://www.lumerical.com.

\section{Acknowledgements}

This work was supported by the National Natural Science Foundation of China $(60907003,61805278,61661004)$, the Guangxi Science Foundation (2017GXNSFAA198227), the Foundation of NUDT (JC13-02-13, ZK17-03-01), the Hunan Provincial Natural Science Foundation of China (13JJ3001), and Program for New Century Excellent Talents in University (NCET) (NCET-12-0142).

\section{Author contributions}

Z.L. and G.L. contributed equally to this work. Z.L. and G.L. finished the algorithm and English writing of the paper together; Z.L. designed and performed the analysis, and prepared the figures/tables; J.Y., J.H., C.Y., Y.Q. and W.X. contributed to manuscript preparation; J.Y. and J.H. conceptualized and coordinated the study; J.Y. and Z.Z. supervised the project. H.H. participated in the revision of the paper.

\section{Competing interests}

The authors declare no competing interests.

\section{Additional information}

Supplementary information is available for this paper at https://doi.org/10.1038/s41598-020-68936-w.

Correspondence and requests for materials should be addressed to Z.Z. or J.Y.

Reprints and permissions information is available at www.nature.com/reprints.

Publisher's note Springer Nature remains neutral with regard to jurisdictional claims in published maps and institutional affiliations.

(c) (i) Open Access This article is licensed under a Creative Commons Attribution 4.0 International License, which permits use, sharing, adaptation, distribution and reproduction in any medium or format, as long as you give appropriate credit to the original author(s) and the source, provide a link to the Creative Commons license, and indicate if changes were made. The images or other third party material in this article are included in the article's Creative Commons license, unless indicated otherwise in a credit line to the material. If material is not included in the article's Creative Commons license and your intended use is not permitted by statutory regulation or exceeds the permitted use, you will need to obtain permission directly from the copyright holder. To view a copy of this license, visit http://creativecommons.org/licenses/by/4.0/.

(c) The Author(s) 2020 